\title{
POSTURE CONTROL AND TRAJECTORY FORMATION DURING ARM MOVEMENT ${ }^{1}$
}

\author{
E. BIZZI, ${ }^{2}$ N. ACCORNERO, ${ }^{3}$ W. CHAPPLE, ${ }^{4}$ AND N. HOGAN ${ }^{5}$ \\ Massachusetts Institute of Technology, Department of Psychology, Cambridge, Massachusetts 02139
}

Received December 12, 1983; Revised May 29, 1984; Accepted May 30, 1984

\begin{abstract}
One hypothesis for the generation of spatially oriented arm movements by the central nervous system is that a desired joint position is determined by the ratio of the tensions of agonist and antagonist muscles. According to this hypothesis, the lransition between equilibrium states should be solely a function of the contraction time of the motor units and the mechanical properties of the arm. We tested this hypothesis in intact and deafferented rhesus monkeys by holding the forearm and measuring the accelerative transient after release of the forearm and by directly measuring the time course of the increase in torque during the movement. Both methods indicated an average time of $400 \mathrm{msec}$ for attaining peak torque in a movement with a duration of $700 \mathrm{msec}$. In addition, by displacing the arm from its normal trajectory during the movement, we observed that the arm returned neither to the initial nor to the final equilibrium positions, but to points intermediate between them. We conclude that the processes underlying trajectory formation must be more complex than a simple switch between one equilibrium position and another.
\end{abstract}

A major problem in the study of motor control has been elucidation of the mechanisms directing the arm to a new spatial location. A distinction is often made (Brooks et al., 1973; Desmedt and Godaux, 1978; Ghez and Vicario, 1978; Desmedt, 1981) between rapid movements, which are believed to be generated by a pulsatile contraction of agonist muscles, and slower ramp movements, which depend upon the smooth contraction of these muscles (Ghez and Vicario, 1978). In both cases, a complex pattern of muscle activation would be required to generate the torques about the joints impelling the arm to the desired position. Feldman (1966) and others (Nichols and Houk, 1976; Kelso, 1977; Cooke, 1979; Kelso and Holt, 1980) have suggested that a muscle is mechanically analogous to a spring, whose stiffness is a function of its activation. As with a spring, a muscle's force is a function of its length. The position at which the length-dependent forces due to opposing muscles are equal is an equilibrium position of the limb. Consequently, the central nervous system (CNS) may maintain a desired joint position by simultaneous activation of agonist and antagonist muscles. This view of posture, in its simplest formulation,

\footnotetext{
${ }^{1}$ This research was supported by National Institute of Neurological and Communicative Disorders and Stroke Research Grant NS09343, National Institute of Arthritis, Metabolism, and Digestive Diseases Grant AM26710, National Aeronautics and Space Administration Grant NAG 2-126, and National Eye Institute Grant EY02621.

${ }^{2}$ To whom correspondence should be addressed.

${ }^{3}$ Present address: University of Rome, Clinica delle Malattie Nervose e Mentali, Rome, Italy.

${ }^{4}$ Present address: University of Connecticut, Physiology Section, Storrs, CT 06268.

${ }^{5}$ Present address: Department of Mechanical Engineering, Massachusetts Institute of Technology, Cambridge, MA 02139.
}

implies that each joint position is coded in the CNS by a single scalar quantity, the ratio of agonist and antagonist forces (Lestienne et al., 1981).

Experimental studies of visually triggered head and arm movements in trained monkeys (Bizzi et al., 1976; Polit and Bizzi, 1979) have shown that a final head and/or forearm position is indeed an equilibrium point between opposing forces. In both normal animals and those deprived of proprioceptive and cutaneous information, brief perturbations of the head or arm initial position by torque pulses did not prevent attainment of the target position, even in the absence of visual feedback. It should be stressed that, in these experiments, as well as in similar studies by Schmidt and McGown (1980), Kelso (1977), and Kelso and Holt (1980), the imposed mechanical disturbances were fairly large and, more importantly, were applied during large movements. These observations led to a control hypothesis that is usually referred to as "final position control." According to this hypothesis, the CNS controls simple large movements by specifying only the final equilibrium point, and the details of movement trajectory are determined by the inherent inertial and viscoelastic properties of the limb and the muscles. In addition, it was proposed that the CNS could define a final limb position by setting the spring constants of agonist and antagonist muscles even in the absence of peripheral feedback. These results were obtained in experiments employing large arm movements with undemanding requirements on final position accuracy. Recent studies have added new dimensions to this work. For instance, it has been shown that disturbances delivered during the execution of fine motor tasks resulted in errors of final position (Sanes and Evarts, 1983). This finding is consistent with the observation that muscle and cutaneous receptors are most sensitive to signals of small amplitude (Matthews and Stein, 1969). Moreover, data reported by Day 
and Marsden (1982) and Rothwell et al. (1982) have indicated that deafferented thumb flexion movements made against a viscous load undershot the intended final position. This observation shows that sensory feedback, in addition to centrally specified final position control, is the mechanism that is used. To interpret these results, we should consider that the distal phalange of the thumb has low inertia and high viscous properties. It is then arguable that the control of the thumb is different from that of other limbs characterized by larger inertia and spring-like behavior.

Regardless of the degree to which peripheral feedback is involved in attaining a final position, the question of how the CNS plans the trajectory of the movement remains.

'Ihe experiments in monkeys reported here were designed to determine whether the trajectories of simple forearm movements, involving one joint and one degree of freedom, are generated by "final position control." Our results indicate that the transition from one arm posture to another is achieved by adjusting the relative intensity of neural signals to each of the opposing muscles so that the equilibrium point defined by their interaction moves toward either flexion or extension of the limb. According to this view, arm trajectory is obtained through neural signals which specify a series of equilibrium positions for the limb.

\section{Materials and Methods}

Movements of the right forearm were studied in three adult rhesus monkeys (Macaca mulatta) before and after surgical elimination of afferent input from the arm. During experimental sessions, the monkey sat in a primate chair with its right arm strapped to a splint, which was clamped to a manipulandum allowing rotation of the forearm about the elbow in the horizontal plane. Ten green, light-emitting diode (LED) targets subtending $2^{\circ}$ were positioned at $10^{\circ}$ intervals in an arc centered on the axis of rotation of the elbow. The monkeys were trained to point to whichever light was on and to hold the arm in that position for an interval of time determined by the experimenter $(1 \mathrm{sec})$. Normally, target lights requiring flexed and extended positions were alternately activated. A trial began with the appearance of a target light. Entry of the monkey's forearm into the electronically defined target window extending $7.5^{\circ}$ on either side of the LED activated a timing circuit. If the forearm remained in the target window until the end of the timing interval (i.e., $1 \mathrm{sec}$ ), the monkey received a reward of water. Attempts at reducing the window size in trained deafferented animals were unsuccessful, because the animals displayed frustration and refused to perform the task. We made no attempt to control the speed of the forearm movements.

Arm movements were monitored by means of a precision potentiometer. A torque motor, coupled to and concentric with the shaft of the splint on which the elbow rested, was used either in a servo or force mode (see below). Strain gauges mounted on the shaft permitted precision measurement of the imposed force, and an accelerometer attached to the splint measured angular acceleration. Muscle activity was monitored by means of Teflon-coated wires implanted in the biceps and the long and medial heads of the triceps. These surgically implanted bipolar electrodes were viable for 1 to 2 months. Subsequently, we implanted pairs of wires percutaneously in flexor and extensor muscle groups immediately before the experimental session. This procedure did not allow us to verify electrode placement in individual flexors and extensors but did permit us to sample the flexors and extensors many times and in different portions of the muscles to ensure the absence of stretch reflexes. At the end of each experiment, known angular displacements and torques were recorded for calibration. To calibrate the accelerometer, we oscillated the manipulandum by feeding a $5-\mathrm{Hz}$ sinusoid from a signal generator through the torque motor. The acceleration was thus the product of the peak-to-peak angular displacement and the square of the angular velocity. Experimental data were recorded on an eight channel chart recorder (Visicorder) and an instrumentation tape recorder (Ampex).

The experiments were conducted in a dark room, and an opaque cover prevented the monkey from seeing its right forearm. However, before each experiment with a deafferented monkey, the monkey was allowed to practice the pointing task for a few minutes with the opaque cover removed.

\section{Deafferentation}

After we had completed a series of experiments in intact monkeys, we performed in each animal a bilateral cervical and upper thoracic $\left(\mathrm{C}_{2}-\mathrm{T}_{3}\right)$ dorsal rhizotomy to eliminate afferent input from the arm. The operation was performed intradurally under a dissecting microscope. We tested the completeness of the dorsal root section by repeatedly looking for a stretch reflex change in the electromyographic (EMG) activity of flexors and extensors when the elbow joint was rapidly extended or flexed through the activation of the torque motor. After a short recovery period ( 2 to 5 days, depending on the condition of the monkey), the monkey's arm movements were studied under the same conditions as before the operation. In these trials, the reward of water was the monkey's only feedback on its performance of the pointing task.

\section{Experimental strategies}

Holding action in the initial position. This experiment was performed in both intact and deafferented monkeys. A target was presented and a position servo circuit (pseudoderivative feedback servomechanism with a limited integrator) (Phelan, 1977), activated by the onset of voluntary EMG, held the forearm in the initial position for various durations. Individual holding actions were applied in a random way. The accleration at the moment of release was solely a function of the torque generated by agonist and antagonist muscles and the inertia of the moving limb. We measured the accelerative transient after release of the arm (Fig. 1) and plotted it against the duration of the holding torque at the initial position. Release of the arm was necessary to demonstrate that the arm achieved the target position under these conditions.

As a complement to this experiment, in deafferented monkeys only, we recorded on the strain gauge the increase in isometric torque during the holding period. Again, holding trials were presented in a random way. If the transition between the initial and final equilibrium positions is rapid and discrete, the profile of torque should reach a constant value within the contraction time of the muscles. On the other hand, should the transition require a much longer time, this would argue for a gradual shift in the torque ratio. We did not perform this experiment in intact animals because, under isometric conditions, torques specified by central commands, and possible reprogramming of these, would have modified the development of torque generation in a way that would have prevented us from observing the time course of centrally specified torques alone.

Holding action in the target position. In the deafferented monkey, an experiment equivalent to "assisting torque pulse" (see below) was

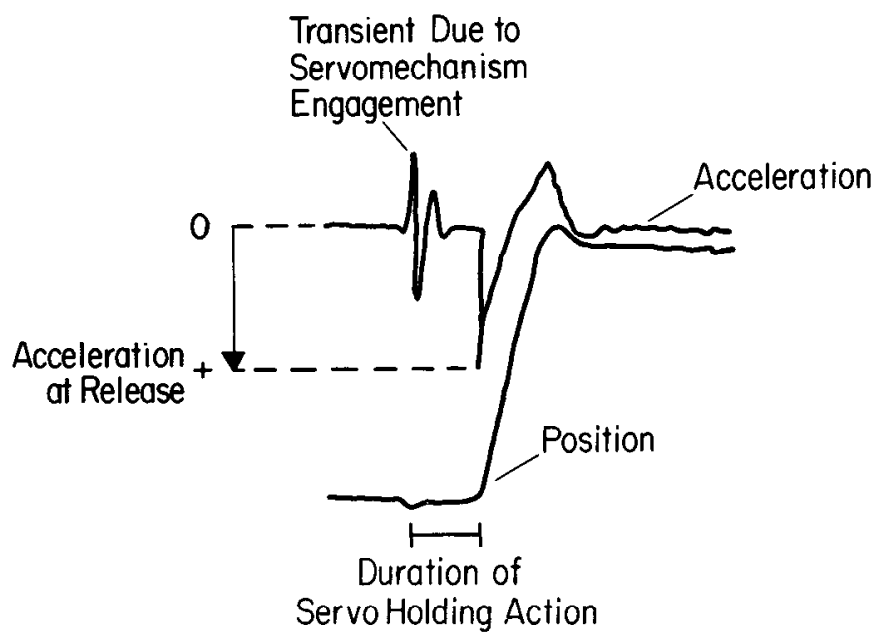

Figure 1. Typical observation from hold-release experiment showing duration of holding action and release. The acceleration plotted in Figure 3 is the value at the moment of release shown above. Positive acceleration is in the downward direction; the arrow in the acceleration trace indicates the measured variable. In all cases, the acceleration was measured with respect to base line. 
performed in which the arm was moved to the final position and then released at different times after EMG onset.

Randomly delivered assisting torque pulse. In the intact monkey, as the forearm began to move toward the target position, a brief torque pulse, generated by injecting a current of known magnitude and duration into the motor, was delivered to the arm in the direction of the movement. The brief torque pulse toward the final position hastened the arrival of the arm to the target. The pulse was triggered by the initial increase of EMG in the agonist muscle.

\section{Results}

We chose to analyze large forearm movements of $60^{\circ}$ in amplitude. The movements usually had smooth trajectories and lasted approximately $692+67 \mathrm{msec}$. Their accelerative and decelerative phases were roughly symmetrical; the average peak absolute acceleration was $1130^{\circ} / \mathrm{sec}^{2} \pm 320^{\circ}(\mathrm{SD} ; n=19)$ for $60^{\circ}$ movements. Most of the movements were somewhat slower than the "continuous" movements studied by Brooks et al. (1973), although some reached peak accelerative values in the ranges they reported. EMG records showed an initial burst in the agonists, followed by a decline to a tonic discharge. For the faster movements, deceleration was accompanied by a short burst in the antagonists. The absence of a second agonist burst provides additional evidence that the movements were not "fast" (Desmedt and Godaux, 1978; Ghez and Vicario, 1978). The fact that our animals were not trained to generate very fast movements (usually characterized by a triphasic EMG pattern) naturally limits our conclusions to a class of mediumspeed movements. It is conceivable that other classes of movement, either the fast ballistic or the smooth pursuit, may be programmed in a different way.

To study the relationship between movement amplitude and stiffness at the termination of movement, small $\left(20^{\circ}\right)$ as well as large $\left(60^{\circ}\right)$ forearm motions were analyzed. (Peak acceleration of $20^{\circ}$ movements was $396.7 \pm 114^{\circ} / \sec ^{2}(n=19)$.)

Holding action in the initial position. This experiment was performed in both intact and deafferented monkeys. When the monkey's arm was briefly held in its initial position after a target had been presented, movements to the target after release of the arm were faster than control movements in the absence of a holding action. The amplitude of the accelerative transient after release of the arm gradually increased with the duration of the holding period (Fig. 2). Figure 3 shows a plot of the accelerative transients against the durations of the holding period in the same animal before and after deafferentation. The time course of the increase in the amplitude of the accelerative transient was virtually identical in the two conditions. Individual holding actions were applied in a random way.

The torque generated by the muscles during the holding period at the initial position was monitored in deafferented animals (Fig. 4). Figure 4, $B$ and $C$, shows two movements with a holding period of about three-quarters of a second, which was longer than the time course of a normal movement to the same target. Upon release, the arm moved to the target position. The peak torque was reached between 350 and $500 \mathrm{msec}$ after the onset of the EMG activity. Table I summarizes the results of three experiments on the development of peak torque in movements of $30^{\circ}$ and $60^{\circ}$ amplitude.

Assisting torque pulse. In the intact monkey, as the forearm began to move toward the target position, a brief torque pulse, whose onset was triggered by the initial increase of EMG in the agonist muscles, was delivered to the arm in the direction of the movement. An assisting torque pulse lasting about 150 msec drove the arm quickly toward the target position. From this position, the forearm returned to a point intermediate between the initial and target positions before moving back to the target position (Fig. 5). As the arm resumed movement toward the target position, its velocity was lower than during control movements to the same target.

Holding action in the target position. When the arm of a
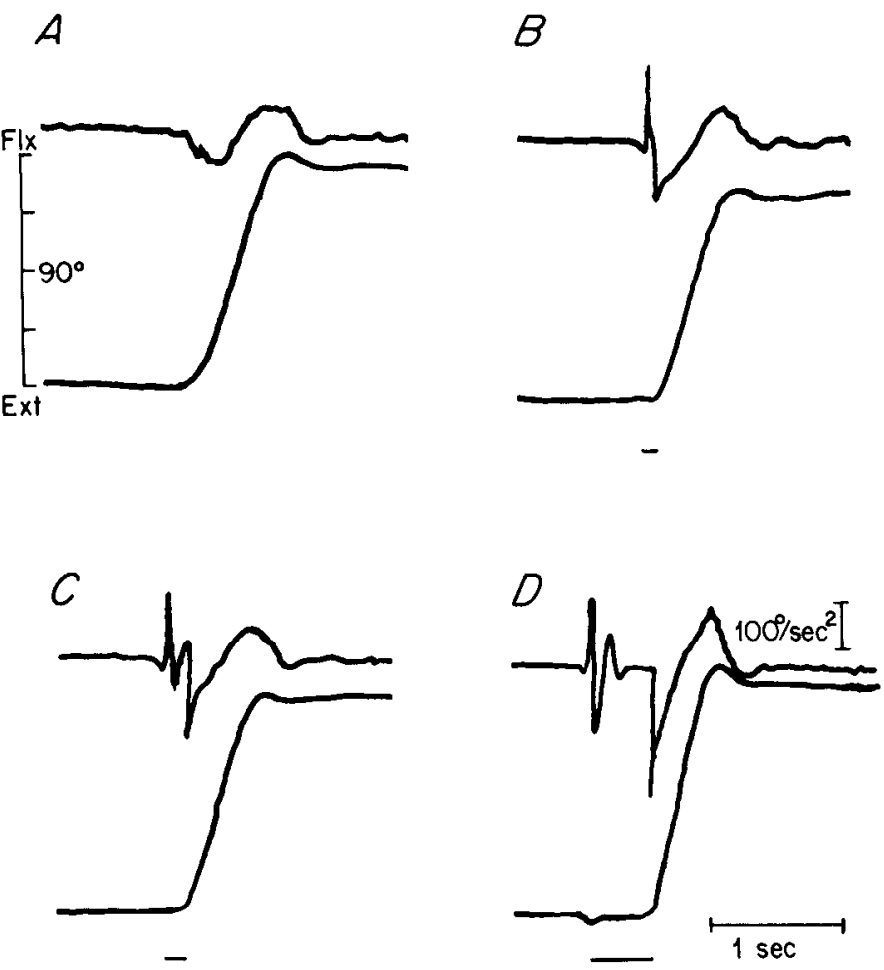

Figure 2. Forearm movements in deafferented monkeys with a holding torque at the initial position. A target was presented, and the arm was held for various durations at the initial position by servomechanism activated by the onset of flexor EMG. The upper trace shows angular acceleration; the lower trace shows arm position. The bar beneath the position trace indicates duration of the holding torque. An elbow angle of $90^{\circ}$ corresponds to the midpoint of the position scale. $A$, Control movement without a holding torque. $B$ to $D$, Movements with holding torque of increasing duration. As the duration increased, the accelerative transient at the beginning of the movement increased. Position scale representing angular excursion $=60^{\circ}$.

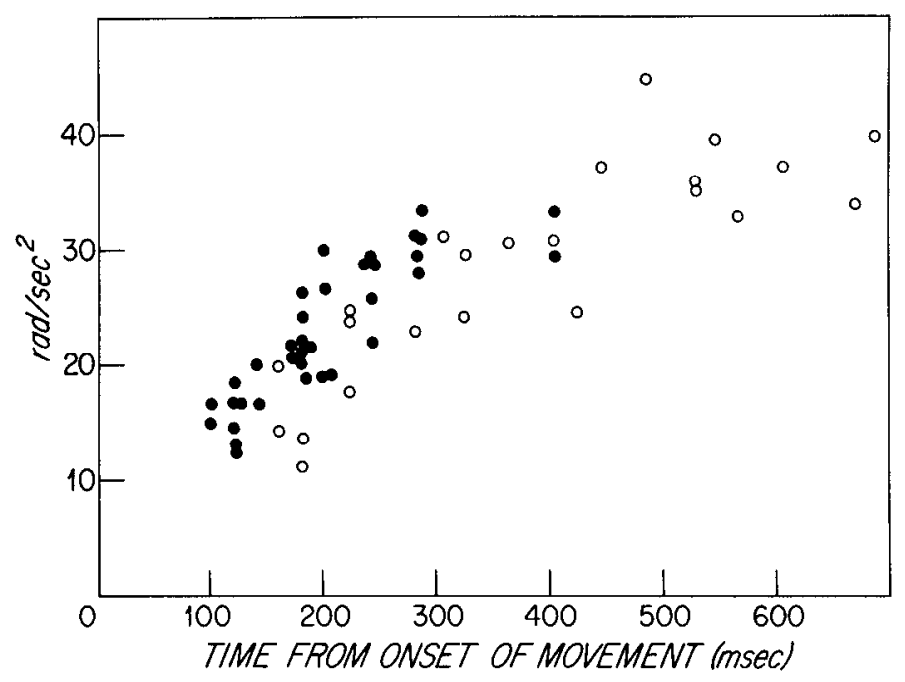

Figure 3. The forearm of intact and deafferented animals was held in its initial position while the animal attempted to move toward a target light and released at various times. This figure is a plot of acceleration immediately following release versus holding time. The abscissa shows time in milliseconds; the ordinate shows radians per second squared. Solid circles, intact animal; open circles, deafferented animal.

deafferented monkey was moved under servo control to a new position, held there for 1 to $2 \mathrm{sec}$ without activation of the target light, and then released, the arm returned to its initial position. However, if the target light at the new position was 


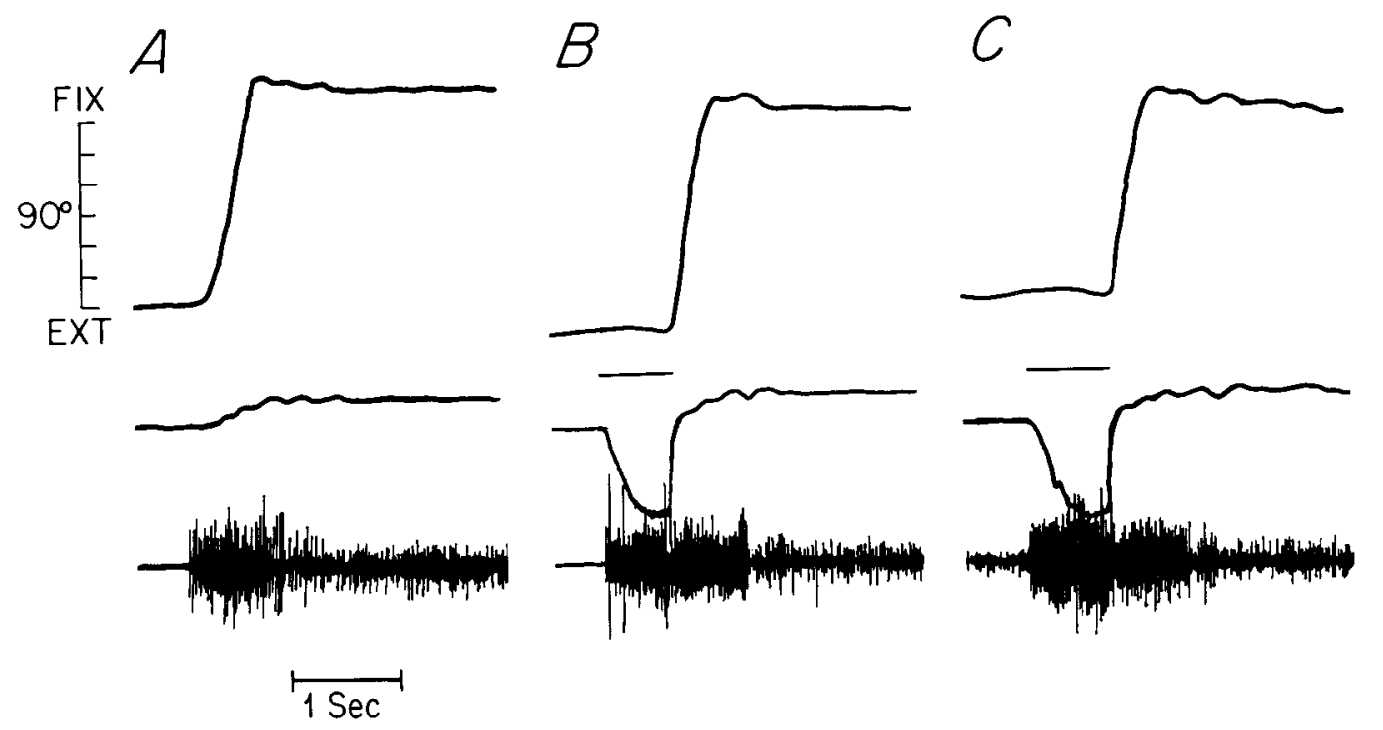

Figure 4. The torque generated while the arm of a deafferented monkey was held at the initial position after a target had been presented. The upper trace shows arm position, the middle trace shows torque, and the lower trace shows flexor (biceps) EMG. The bar beneath the arm trace represents duration of the holding torque. $A$, Control movement without a holding torque. $B$ and $C$, Two movements in which the buildup of isometric tension can be observed. Position scale representing angular excursion $=60^{\circ}$.

TABLE I

Time course of isometric torque in deafferented animals The experimental paradigm is that of Figure 4. Latency of peak force was measured from the initial EMG burst to the point in the torque profile at which the torque was constant.

\begin{tabular}{cc}
\hline $\begin{array}{c}\text { Time Course to Peak Force } \\
\text { from Start of EMG }\end{array}$ & Peak Force \\
\hline$m s e c$ & $m N-m^{a}$ \\
$30^{\circ}$ Amplitude & \\
$480 \pm 66(n=10)$ & $337 \pm 123$ \\
$366 \pm 72(n=16)$ & $252 \pm 70$ \\
$400 \pm 147(n=15)$ & $378 \pm 76$ \\
$\quad 60^{\circ}$ Amplitude & \\
$488 \pm 92(n=16)$ & $507 \pm 56$ \\
\hline
\end{tabular}

${ }^{a} \mathrm{mN}-\mathrm{m}$, millinewton-meters.

activated while the servo kept the arm in place, a new set of events was observed (Fig. 6). When the usual reaction time after the appearance of a target had elapsed, the monkey activated its muscles (flexors in the case of Fig. 6) to acquire the target position. At this point, usually shortly after the onset of muscle activity, the servo was deactivated. The arm then returned to a point intermediate between the initial and the target positions before moving back to the target position. Note that, during the return movement requiring extension, evident flexor activity was present. The amplitude of the return movement was a function of the duration of the holding action. If enough time (at least $1 \mathrm{sec}$ ) elapsed between activation of the target light and deactivation of the servo, the arm remained in the target position upon release. These results were obtained in many trials during several sessions with two monkeys.

Final equilibrium stiffness. The simplest model for the generation of pointing movements assumes that the stiffness about the final equilibrium position is independent of the amplitude of the movement to that position. Hence, we measured the stiffness after movements of different amplitudes to the same position. However, we found that the final equilibrium stiffness varied with the amplitude of the movement and that the CNS may therefore take amplitude into account. Table II summarizes the results of four experiments in two normal monkeys and one experiment in a deafferented monkey. There was a small but statistically significant difference in the stiffness about the final position after large $\left(60^{\circ}\right)$ - and small-amplitude movements $\left(20^{\circ}\right)$ to the same target, and a much greater variation in the stiffness after movements of the same amplitude made on different days. The stiffness after the movement was much lower in the deafferented monkeys than in the normal ones; indeed, the stiffness was so low in some animals that it was difficult to obtain an equilibrium value.

It should be emphasized that we measured the stiffness only at the final position. Although we attempted to measure stiffness during the course of the movement, the mechanical conditions at this time are much more complex and it proved difficult to unambiguously dissociate the component of net torque resulting from muscle stiffness from components reflecting the inertia and viscosity of the moving arm.

\section{Discussion}

Previous work has shown that monkeys can execute simple single-joint pointing movements of the forearm and maintain briefly ( $1 \mathrm{sec}$ ) the forearm in a new equilibrium position in the complete absence of proprinceptive feedback from forearm muscles and joints (Polit and Bizzi, 1979). These findings imply a functional relationship between the descending commands to the relevant muscles and the equilibrium position of the forearm. Hence, in the present study, we addressed the question of whether a single-joint pointing movement of the forearm in the monkey can be described merely as a shift of posture. If this were the case, we would expect to observe a rapid shift to the final equilibrium position, and the time course of the movement would be determined by the dynamics of muscle contraction, limb inertia, and viscosity. Our findings suggest instead that these simple forearm movements do not result from rapid shifts in the equilibrium point. It should be stressed that in this series of experiments we have focused exclusively on large, low accuracy forearm movements and that we did not make any attempt to control the speed of forearm movements by inducing, through training, very fast responses to target presentation. Consequently, the significance of our observations cannot safely be extended to other conditions such as high accuracy, small movements (Sanes and Evarts, 1983) or movements of segments which, like the thumb, are characterized by different mechanical conditions (Day and Marsden, 1982; Rothwell et 

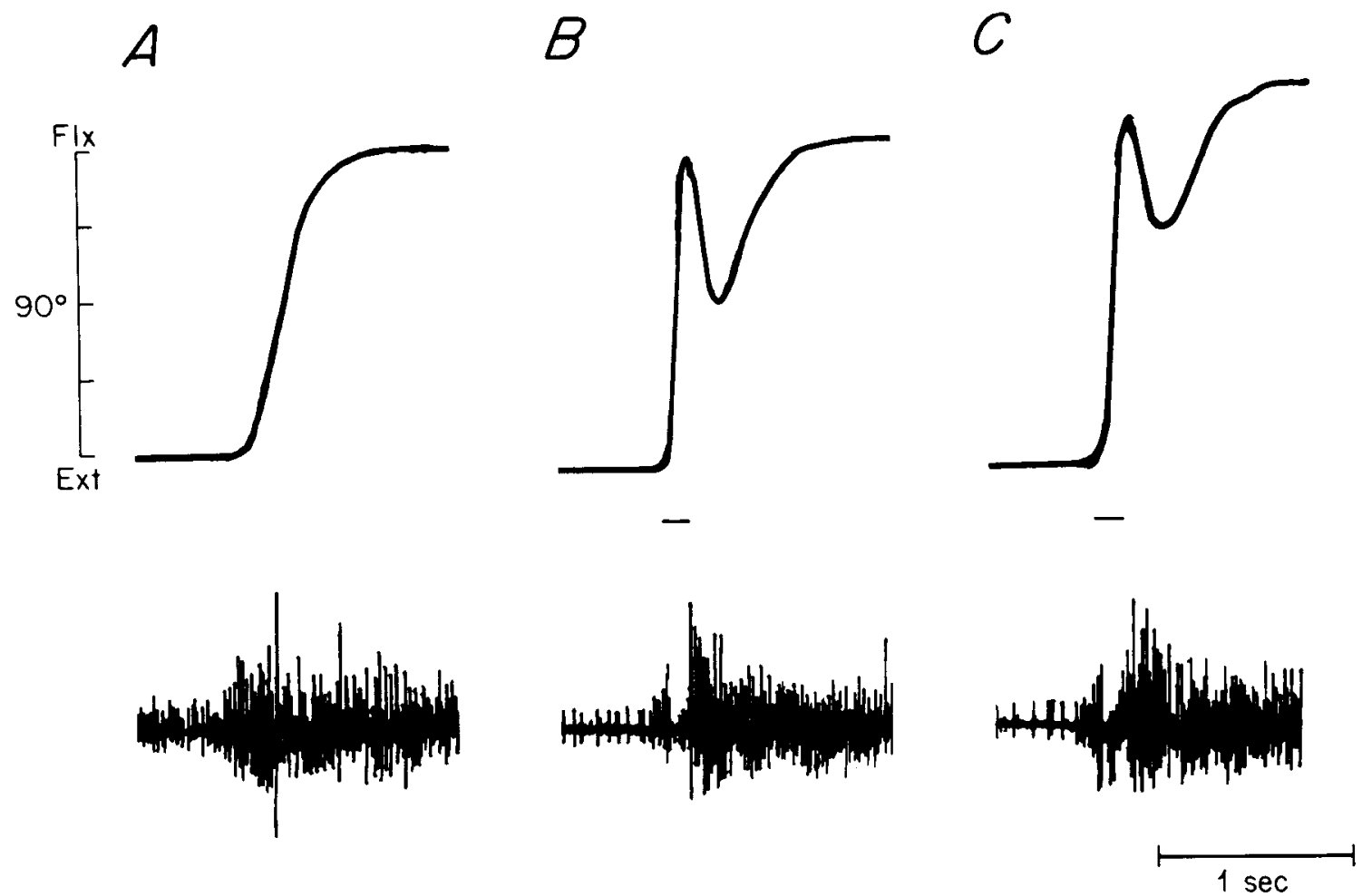

Figure 5. Forearm movements with an assisting torque pulse in an intact animal. The upper trace shows arm position with an elbow angle of $90^{\circ}$ at the midpoint of the scale; the lower trace shows flexor (biceps) EMG. The bar beneath the position trace indicates duration of the torque pulse. $A$, Control movement without a torque pulse. $B$ and $C$, Two movements with torque pulses. The arm reached the target position early in the movement, transiently returned to an intermediate position, and then moved back to the target position. Note the unloading reflex in the EMG trace. Flx, flexion; Ext, extension. Position scale representing angular excursion $=60^{\circ}$.

al., 1982). For example, in contrast to the arm, the posture of the distal phalange of the thumb is insensitive to the orientation of the hand in the gravitational field (even when the muscles are relaxed). Indeed, the mechanical conditions of the thumb more closely resemble those of the eye than of the hand. It is then entirely possible that in these situations, different control schemes may be operative.

Critique of the hypothesis of "final position control": Theoretical considerations. According to the hypothesis of "final position control," we would expect the steady-state equilibrium position to be achieved after a delay due to the dynamics of muscle activation; i.e., twitch contraction time. To estimate this dynamic effect, we made the worst theoretical case assumption that all of the motor units recruited have a twitch contraction time corresponding to the mean value of $50 \mathrm{msec}$ (Buchthal and Schmalbruch, 1970; Collatos et al., 1977). A simple summation of these twitches (corresponding to the summation of motor unit tensions in the tendon) yields a net muscle force which rises to within a few percent of the final value within $200 \mathrm{msec}$ (Hogan, 1984).

Experimental results. We found that, for a $60^{\circ}$ movement lasting $600 \mathrm{msec}$ or more, the torque produced by the alpha motoneuronal activity did not reach steady state until $400 \mathrm{msec}$ or more had elapsed after the onset of action potentials in the muscle. This was clearly seen when a target was presented and the torque motor used in a servo mode held the arm at the initial position for various durations (Fig. 3). The initial acceleration after release of the arm increased gradually with the duration of the holding period, reaching a steady-state value no sooner than $400 \mathrm{msec}$ after the onset of EMG activity (Fig. 3 ). Equivalently, the torque generated in response to alpha motoneuronal activity during the holding period at the initial position increased gradually with time, reaching a peak for $60^{\circ}$ movements at $488 \mathrm{msec}$ (average value) after the onset of EMG activity (Fig. 4, Table I). Taken together, these results show that the CNS had programmed a slow, gradual shift of the equilibrium point, a fact which is not consistent with the "final position control" hypothesis.

Our findings when the forearm was quickly forced to the target position by an assisting torque pulse applied at the beginning of the movement (normal animals), or when it was moved to the target position under servo control and then relcased from that position after the onset of EMG activity (deafferented animals), were also inconsistent with the hypothesis of final position control. In the first case, the forearm returned to a point between the initial and final target positions before proceeding to the endpoint. Because the animal was intact, one could argue that the return movements could be due to reflex activation of the muscles stretched by the action of the assisting pulse combined with the unloading of the agonist (see Fig. 5). Given this situation, we performed, in the deafferented animal, the experiment described as "holding action" in the target position. Again, we observed that the forearm returned to a point halfway, but in this case the agonist muscle unmodulated by the action of reflexes was active throughout the to and fro motion shown in Figure 6. These observations suggest that the alpha motoneuronal activity specifies not only a position for the forearm at equilibrium, as previously shown (Polit and Bizzi, 1979), but also a series of equivalent equilibrium positions throughout the movement. If the muscles merely generated force during the transient phase of a movement, we would not have seen the pronounced return motion of the limb during flexor muscle activity (Fig. 6). However, it is well known that the force generated by a muscle is a function of its length and that the torque generated by a group of muscles is a function of the angles of the corresponding joints. As a direct result of this position dependence, the alpha activities of the muscles can always be interpreted as specifying an equilibrium 


\section{Servo Action}

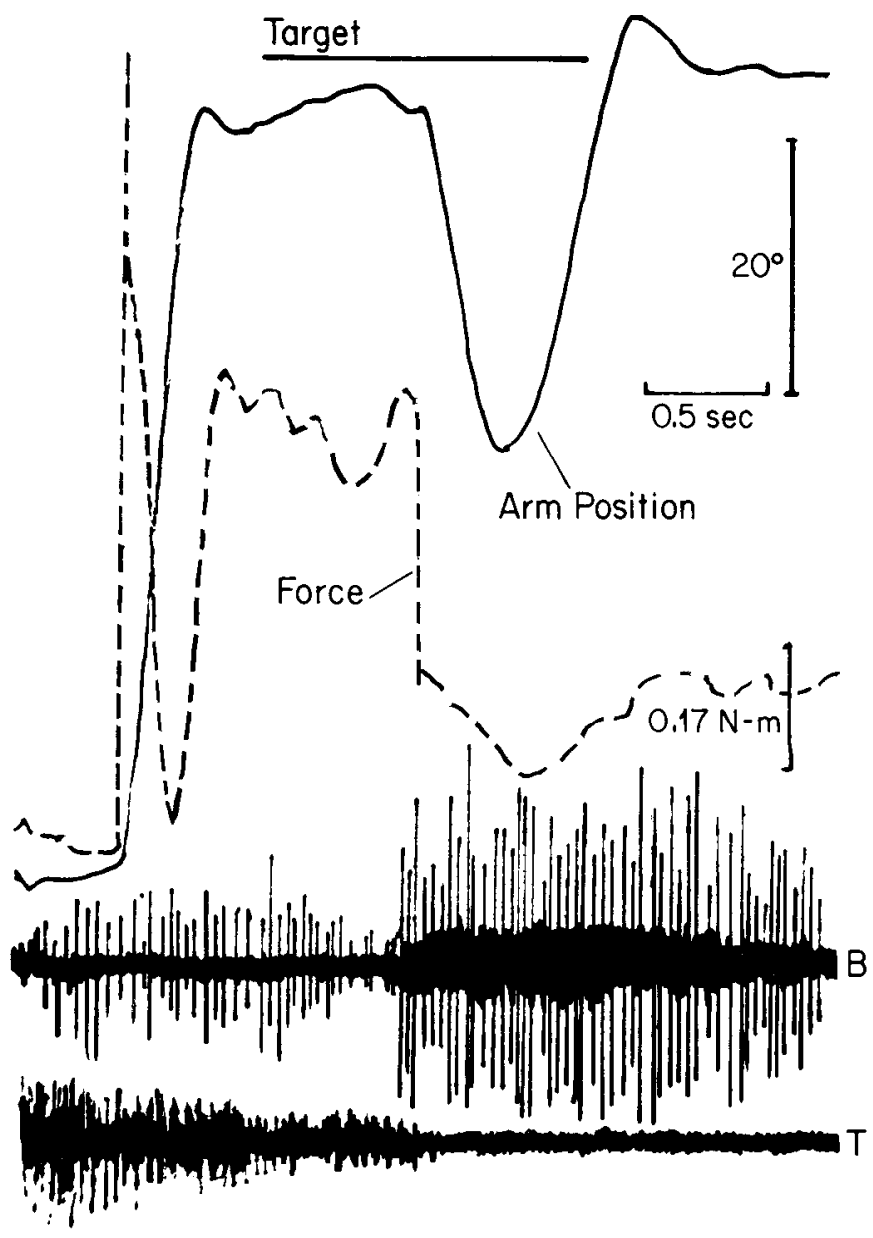

Figure 6. Forearm movements of deafferented monkeys with a holding action in the final position. While the target light remained off, the servo moved the arm to the target position, the target light was activated, and the servo was turned off. The arm returned to a position intermediate between the initial and target positions before moving back to the target position. Similar results were obtained in many trials in two monkeys. The upper bar indicates duration of servo action. The lower bar indicates onset of the target light. The broad trace shows arm position; the dashed trace shows torque. $B$, flexor (biceps); $T$, extensor (triceps).

position, along with a stiffness about this position. Thus, in the transition from the initial to the final limb position, the alpha motoneuronal activity is defining a series of equivalent equilibrium positions. (We term this the "virtual position," and its time history the "virtual trajectory" (Hogan, 1981).) As a result, after cessation of an assisting torque pulse or a holding action in the final position, the forearm headed for a position intermediate between the initial and final positions.

It must be stressed that the dependence of muscle force on muscle length means that alpha motoneuronal activity can be interpreted as commanding either a limb position, with the stiffness specifying a force corresponding to the difference between the actual and the commanded positions, or a force, with the stiffness specifying the position corresponding to the difference between the actual and the commanded forces. A question of long-standing interest in the neurophysiology of motor control has been whether a descending command specifies a position or a force. If the characteristics of muscle are such that force and length are uniquely related, then the two are mechanically equivalent and indistinguishable. In postulat- ing that forearm trajectory is determined by a series of positions or forces, we have applied known length-tension relationships derived under static conditions to a dynamic situation. $\Lambda \mathrm{l}$ though such factors as the relationship between force and velocity and the history of muscle activation may alter the length-tension relationships during movement, we feel that the static extrapolation provides insight into our results (Hogan, 1984).

Our comparison of forearm movements in intact and deafferented monkeys revealed some quantitative differences, but no qualitative differences. This finding indicates that sensory feedback was unnecessary for implementation of the fundamental strategy underlying these movements. In the context of these experiments, the major role of the feedback was to augment inherent properties of the muscle, such as stiffness. In the highly trained and deafferented animal, the simple task of moving only one joint was executed primarily by a movement program of central origin.

A question we have not resolved is what central processes other than the programming of a "virtual trajectory" are involved in moving from the initial to the final position. Our measurements of large- and medium-amplitude movements to a final position showed in intact animals a small but significant difference in final stiffness, perhaps because of greater cocontraction of agonist and antagonist muscles with greater amplitudes of movement. This result indicates that specification of the final equilibrium point involves more than setting the ratio of the agonist and antagonist muscle activities.

Finally, it should be stressed that the forearm movements we studied were performed at moderate speed. It is conceivable that, for very fast movements, the shift in equilibrium point may be more abrupt (step-like) or may even specify a point beyond the intended equilibrium point (Hogan, 1984), which would amount to a pulse-step command of the type known to control eye movements (Robinson, 1970) and fast limb movements (Desmedt and Godaux, 1978).

Although we analyzed movements about a single joint, the trajectories of multi-joint movements may also be generated by a gradual shift in equilibrium point. Because of the complex kinematics and dynamics of multi-joint movement, a step-like transition might gencrate forces difficult to control. The gradual transition between equilibrium positions, however, would facilitate coordination of multi-joint movement and reduce the problem of control.

TABLE II

Stiffness $0.5 \mathrm{sec}$ after the end of movement

Torque pulses of approximately $0.1 \mathrm{~N}$-m amplitude and $0.5 \mathrm{sec}$ duration directed toward extension were used to deflect the arm. The consequence deflection was recorded. The $t$-test was calculated for the hypothesis that the stiffness at the end of small and large amplitude movements was different. The tests on $1 / 14 / 80,1 / 15 / 80$, and $1 / 20 / 80$ were significant at the $0.5 \%$ level, and the test on $1 / 17 / 80$ was significant at the $5 \%$ level. For comparative purposes, stiffness of a deafferented preparation is shown.

\begin{tabular}{|c|c|c|}
\hline \multirow{2}{*}{ Date } & \multicolumn{2}{|c|}{ Stiffness } \\
\hline & Small Amplitude & Large Amplitude \\
\hline $1 / 14 / 80$ & $\begin{aligned} 1.50 & \pm 0.16\left(20^{\circ}\right) \\
n & =9\end{aligned}$ & $\begin{aligned} 2.04 & \pm 0.47\left(60^{\circ}\right) \\
n & =8\end{aligned}$ \\
\hline $1 / 15 / 80$ & $\begin{aligned} 1.21 & \pm 0.17\left(20^{\circ}\right) \\
n & =30\end{aligned}$ & $\begin{aligned} 1.46 & \pm 0.36\left(60^{\circ}\right) \\
n & =20\end{aligned}$ \\
\hline $1 / 17 / 80$ & $\begin{aligned} 1.76 & \pm 0.34 \mathrm{~N}-\mathrm{m} / \mathrm{rad}^{-1}\left(30^{\circ}\right) \\
n & =11\end{aligned}$ & $\begin{aligned} 1.98 & \pm 0.38\left(70^{\circ}\right) \\
n & =10\end{aligned}$ \\
\hline $1 / 22 / 80$ & $\begin{aligned} 1.13 & \pm 0.15 \mathrm{~N}-\mathrm{m} / \mathrm{rad}\left(20^{\circ}\right) \\
n & =15\end{aligned}$ & $\begin{aligned} 1.35 & \pm 0.24 \mathrm{~N}-\mathrm{m} / \mathrm{rad}\left(60^{\circ}\right) \\
n & =15\end{aligned}$ \\
\hline Deafferented & & $\begin{aligned} 0.78 & \pm 0.16 \mathrm{~N}-\mathrm{m} / \mathrm{rad}\left(50^{\circ}\right) \\
n & =18\end{aligned}$ \\
\hline
\end{tabular}




\section{References}

Bizzi, E., A. Polit, and P. Morasso (1976) Mechanisms underlying achievement of final head position. J. Neurophysiol. 39: 435-444.

Brooks, V. B., A. Atkin, M. Uno, I. B. Kozlovskaya, and F. E. Horvath (1973) Fffects of cooling dentate nucleus on tracking-task performance in monkeys. J. Neurophysiol. 36: 974-995.

Buchthal, F., and H. Schmalbruch (1970) Contraction times and fibre types in intact human muscle. Acta Physiol. Scand. 79: 435 452.

Collatos, T. C., V. R. Edgerton, J. L. Smith, and B. R. Botterman (1977) Contractile properties and fiber type compositions of flexors and extensors of elbow joint in cat: Implications for molor control. J. Neurophysiol. 40: 1292-1300.

Cooke, J. D. (1979) Dependence of human arm movements on limb mechanical properties. Brain Res. 165: 366-369.

Day, B. L., and C. D. Marsden (1982) Accurate repositioning of the human thumb against unpredictable dynamic loads is dependent upon peripheral feed-back. J. Physiol. (Lond.) 237: 393-407.

Desmedt, J. E. (1981) The size principle of motoneuron recruitment in ballistic or ramp voluntary contractions in man. Prog. Clin. Neurophysiol. 9: 97-136.

Desmedt, J. E., and E. Godaux (1978) Ballistic skilled movements: Load compensation and patterning of the motor commands. Prog. Clin. Neurophysiol. 4: 21-55.

Feldman, A. G. (1966) Functional tuning of the nervous system during control of movement or maintenance of a steady posture. III. $\mathrm{Me}$ chanographic analysis of the execution by man of the simplest motor tasks. Biophysics 11: 766-775.

Ghez, C., and D. Vicario (1978) The control of rapid limb movement in the cat. II. Scaling of isometric force adjustments. Exp. Brain Res. 33: $191-202$.

Hogan, N. (1984) An organizing principle for a class of voluntary movements. J. Neurosci. 4: 2745-2754.
Kelso, J. A. S. (1977) Motor control mechanisms underlying human movement reproduction. J. Exp. Psychol. 3: 529-543.

Kelso, J. A. S., and K. G. Holt (1980) Exploring a vibratory system analysis of human movement production. J. Neurophysiol. 43: 11831196.

T.estienne, F., A. Polit, and F. Bizzi (1981) Functional organization of the motor process underlying the transition from movement to posture. Brain Res. 230: 121-131.

Matthews, P. B. C., and R. B. Stein (1969) The sensitivity of muscle spindle afferents to small sinusoidal changes of length. J. Physiol. (Lond.) 200: 723-743.

Nichols, T. R., and J. C. Houk (1976) Improvenent in linearity and regulation of stiffness that results from actions of stretch reflex. $J$. Neurophysiol. 39: 119-142.

Phelan, R. M. (1977) Automatic Control Systems, Cornell University Press, New York.

Polit, A., and E. Bizzi (1979) Characteristics of the motor programs underlying arm movements in monkeys. J. Neurophysiol. 42: 183194.

Rack, P. M. H., and D. R. Westbury (1969) The effects of length and stimulus rate on tension in the isometric cat soleus muscle. $J$. Physiol. (Lond.) 204: 443-460.

Robinson, D. A. (1970) Oculomotor unit behavior in the monkey. J. Neurophysiol. 33: 393-404.

Rothwell, J. C., M. M. Traub, B. L. Day, J. A. Obeso, P. K. Thomas, and C. D. Marsden (1982) Manual motor performance in a deafferented man. Brain 105: 515-542.

Sanes, J. N., and E. V. Evarts (1983) Effects of perturbations on accuracy of arm movements. J. Neurosci. 3: 977-986.

Schmidt, R. A., and C. McGown (1980) Terminal accuracy of unexpectedly loaded rapid movements: Evidence for a mass-spring mechanism in programming. J. Motor Behav. 12: 149-161. 